\title{
Calculation of Certain Multiple Generating Functions
}

\section{George H. Weiss}

(September 19, 1963)

\begin{abstract}
This paper contains a discussion of the evaluation of generating functions of the form $F(\{x\})$ $=\sum_{n_{1}} \ldots \sum_{n_{k}} M_{j}\left(n_{1}, \ldots n_{k}\right) x_{1}{ }^{n_{1}} x_{2}{ }^{n_{2}} \ldots x_{k}{ }^{n_{k}}$ where $M_{j}\left(n_{1}, \ldots n_{k}\right)$ is the $j$ th largest of the integers $\left(n_{1}, \ldots n_{k}\right)$. An alternate technique to one proposed by Carlitz is used in the calculation.
\end{abstract}

In a recent paper Carlitz has considered the problem of evaluating the generating functions

$$
F_{j}(\{x\})=\sum_{n_{1}=0}^{\infty} \ldots \sum_{n_{k}=0}^{\infty} M_{j}\left(n_{1}, n_{2}, \ldots n_{k}\right) x_{1}^{n_{1}} x_{2}^{n_{2}} \ldots x_{k_{k}}^{n_{k}}
$$

where $M_{j}\left(n_{1}, n_{2}, \ldots n_{k}\right)$ is the $j$ th greatest of the set of integers $\left\{n_{j}\right\},[1] .{ }^{1}$ His method was essentially a combinatorial one. It is our purpose in the present note to reconsider this problem by evaluating, instead of $F_{j}$, the generating function

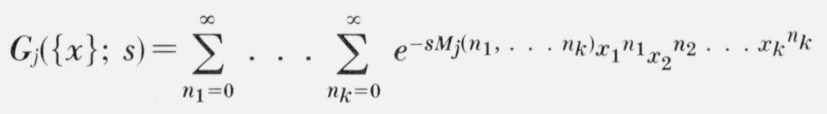

from which it is possible to derive an expression for $F_{j}$ by differentiation

$$
F_{j}(\{x\} ; s)=-\left.\frac{\partial G_{j}}{\partial s}(\{x\} ; s)\right|_{s=0+} .
$$

Expressions for related generating functions can also be obtained in this manner.

The principal tool in the following analysis is the identity

$$
e^{-s M_{1}\left(n_{1}, \ldots n_{k}\right)}=s \int_{M_{1}\left(n_{1}, \ldots n_{k}\right)}^{\infty} e^{-s t} d t=s \int_{0}^{\infty} e^{-s t} H\left(t-n_{1}\right) H\left(t-n_{2}\right) \ldots H\left(t-n_{k}\right) d t
$$

where $H(x)$ is the Heaviside step function

$$
\begin{aligned}
H(x) & =0, x<0 \\
& =1, x>0 .
\end{aligned}
$$

We can immediately derive the expression for $G_{1}(\{x\} ; s)$ from eq (4) by multiplying the general term of this equation by $x_{1}{ }^{n_{1}} \ldots x_{k}{ }^{n_{k}}$ and summing. An interchange of the orders of summation and integration is easily justified for $\left|x_{1}\right|,\left|x_{2}\right|, \ldots\left|x_{k}\right|<1$ and so we have only to evaluate the sum

$$
\sum_{n=0}^{\infty} H(t-n) x^{n}=\sum_{n=0}^{[t]} x^{n}={\frac{1-x^{[t]+1}}{1-x}}
$$

${ }^{1}$ L. Carlitz, The generating function for $\max \left(n_{1}, \ldots n_{k}\right)$, Portugaliae, Mathematica 21, 201 (1962), 
With this result we find

$$
G_{1}(\{x\} ; s)=\frac{s}{\left(1-x_{1}\right)\left(1-x_{2}\right) \ldots\left(1-x_{k}\right)} \int_{0}^{\infty} e^{-s t}\left(1-x_{1}^{[t]+1}\right)\left(1-x_{2}[t]+1\right) \ldots\left(1-x_{k}^{[t]+1}\right) d t .
$$

In order to evaluate this expression we need the following Laplace transform

$$
s \int_{0}^{\infty} e^{-s t} a^{[t]+1} d t=\frac{a\left(1-e^{-s}\right)}{1-a e^{-s}}=\left(e^{s}-1\right)\left(\frac{1}{1-a e^{-s}}-1\right)
$$

where $a$ is a constant. Equations (7) and (8) together yield:

$$
\begin{aligned}
& G_{1}(\{x\} ; s)=\frac{1}{\left(1-x_{1}\right) \ldots\left(1-x_{k}\right)}\left\{1-\left(e^{s}-1\right) \sum_{r}\left(\frac{1}{1-x_{r} e^{-s}}-1\right)\right. \\
&\left.+\left(e^{s}-1\right) \sum_{\substack{r_{1} \\
r_{1}>r_{2}}} \sum_{r_{2}}\left(\frac{1}{1-x_{r_{1}} x_{r_{2}} e^{-s}}-1\right)-\left(e^{s}-1\right) \sum_{\substack{r_{1} \\
r_{1}>r_{2}>r_{3}}} \sum_{\substack{r_{2} \\
r_{1}}}\left(\frac{1}{1-x_{r_{1}} x_{r_{2}} x_{r_{3}} e^{-s}}-1\right)+\ldots\right\}
\end{aligned}
$$

Now let us consider the evaluation of the other $G_{j}$. We require, for these generating functions, identities similar to that of eq (4). The preceding analysis suggests that we try the sum of integrals

$$
\begin{aligned}
s \int_{0}^{\infty} e^{-s t}\left\{\left[1-H\left(t-n_{1}\right)\right] H(t-\right. & \left.n_{2}\right) \ldots H\left(t-n_{k}\right)+H\left(t-n_{1}\right)\left[1-H\left(t-n_{2}\right)\right] H\left(t-n_{3}\right) \ldots H\left(t-n_{k}\right) \\
& \left.+\ldots+H\left(t-n_{1}\right) H\left(t-n_{2}\right) \ldots\left[1-H\left(t-n_{k}\right)\right]\right\} d t=U\left(n_{1}, n_{2}, \ldots n_{k} ; s\right)
\end{aligned}
$$

for the evaluation of $G_{2}$. By repeatedly using eq (4) we find

$$
\begin{aligned}
U\left(n_{1}, \ldots n_{k} ; s\right)=\left[e^{-s M_{1}\left(n_{2}, \ldots n_{k}\right)}-\right. & \left.e^{-s M_{1}\left(n_{1}, \ldots n_{k}\right)}\right]+\left[e^{-s M_{1}\left(n_{1}, n_{3}, \ldots n_{k}\right)}\right. \\
& \left.-e^{-s M_{1}\left(n_{1}, \ldots n_{k}\right)}\right]+\ldots+\left[e^{-s M_{1}\left(n_{1}, \ldots, n_{k-1}\right)}-e^{-s M_{1}\left(n_{1}, \ldots n_{k}\right)}\right] .
\end{aligned}
$$

Assume now that the maximum of the $\left\{n_{j}\right\}$ is $n_{1}$. Then since $n_{1}$ appears in each of the terms in all of the brackets except the first, these brackets must be equal to zero. Furthermore we must have

$$
M_{1}\left(n_{2}, \ldots n_{k}\right)=M_{2}\left(n_{1}, \ldots n_{k}\right)
$$

Hence we find

$$
G_{2}(\{x\} ; s)-G_{1}(\{x\} ; s)=\sum_{n_{1}} \ldots \sum_{n_{k}} U_{1}\left(n_{1}, \ldots n_{k} ; s\right) x_{1}{ }^{n_{1}} \ldots x_{k}{ }^{n_{k}}
$$

The function $G_{1}(\{x\} ; s)$ has already been calculated in eq (9) so that $G_{2}$ can be determined to be

$$
G_{2}(\{x\} ; s)=\sum_{r=1}^{k} G_{1}\left(\{x\}-x_{r} ; s\right)+(1-k) G_{1}(\{x\} ; s)
$$

where $\{x\}-x_{r}$ is the set $\{x\}$ less the element $x_{r}$. Similar expressions can be obtained for all of the $G_{j}$ by starting from a sum of integrals similar to eq (4), each of which has $j$ factors $1-H\left(t-n_{r}\right)$ with the remaining factors being of the form $H\left(t-n_{r}\right)$. In this way we can write the recurrence relation

$$
G_{j+1}(\{x\} ; s)=\sum_{r=1}^{k} G_{j}\left(\{x\}-x_{r} ; s\right)+(1-k) G_{j}(\{x\} ; s) .
$$


The function $F_{1}(\{x\})$ is now obtained from eqs (3) and (9) in a straightforward manner:

$$
\begin{aligned}
F_{1}(\{x\})=\frac{1}{\left(1-x_{1}\right) \ldots\left(1-x_{k}\right)}\left\{1-\sum_{r} \frac{x_{r}}{1-x_{r}}+\sum_{r_{1} \geq r_{2}}\right. & \frac{x_{r_{1}} x_{r_{2}}}{1-x_{r_{1}} x_{r_{2}}}-\ldots \\
& \left.+(-1)^{k} \frac{x_{1} x_{2} \ldots x_{k}}{1-x_{1} x_{2} \ldots x_{k}}\right\}
\end{aligned}
$$

and others of the $F_{j}$ can be derived by recurrence

$$
F_{j+1}(\{x\})=\sum_{r=1}^{k} F_{j}\left(\{x\}-x_{r}\right)+(1-k) F_{j}(\{x\})
$$

By these techniques we may derive Carlitz's result

$$
F_{j}(\{x\})=\sum_{s=j}^{k}(-1)^{s+j}\left(\begin{array}{l}
s-1 \\
j-1
\end{array}\right) \cdot U_{k s}
$$

where

$$
U_{k s}=\frac{1}{\left(1-x_{1}\right)\left(1-x_{2}\right) \ldots\left(1-x_{k}\right)} J \frac{x_{1} x_{2} \ldots x_{s}}{1-x_{1} x_{2} \ldots x_{s}}
$$

where $J f\left(x_{1}, x_{2}, \ldots x_{s}\right)$ is the symmetric function determined by $f\left(x_{1}, x_{2}, \ldots x_{s}\right)$.

Similar techniques can also be used for the calculation of generating functions like

$$
H_{j}(\{x\})=\sum_{n_{1}=0}^{\infty} \ldots \sum_{n_{k}=0}^{\infty} M_{j}\left(n_{1}^{\lambda}, n_{2}^{\lambda}, \ldots n_{k}^{\lambda}\right) x_{1}^{n_{1}} \ldots x_{k}^{n_{k}}=\sum_{n_{1}=0}^{\infty} \ldots \sum_{n_{k}=0}^{\infty}\left[M_{j}\left(n_{1}, n_{2}, \ldots n_{k}\right)\right]^{\lambda} x_{1} n_{1} \ldots x_{k}{ }^{n} k
$$

When $\lambda$ is an integer $H_{j}$ can be expressed as a derivative of $G_{j}$. However, one can calculate $H_{j}$ for any $\lambda$ by the same technique as we have used for $\lambda=1$. Define a function $G_{j}^{(\lambda)}(\{x\} ; s)$ analogous to that in eq (4) except that $M_{j}\left(n_{1}, \ldots n_{k}\right)$ is replaced by $M_{j}\left(n_{1}^{\lambda}, \ldots n_{k}^{\lambda}\right)$. Then eq (4) remains valid except that each $n_{j}$ is to be replaced by $n_{j}^{\lambda}$ and the succeeding steps lead, in the case $j=1$, to the expression

$$
G_{1}^{\lambda}(\{x\} ; s)=\frac{s}{\left(1-x_{1}\right) \ldots\left(1-x_{k}\right)} \int_{0}^{\infty} e^{-s t}\left(1-x_{1}^{\frac{1}{[t \lambda]}} \ldots\left(1-x_{k} \frac{1}{[t \lambda]+1}\right) d t .\right.
$$

The Laplace transform of $a^{\left[t^{\frac{1}{\lambda}}\right]_{+1}}$ is

$$
\left.s \int_{0}^{\infty} e^{-s t}{ }_{a}{ }_{t}{ }_{t}^{1}\right]_{+1} d t=a\left[1-(1-a) \sum_{n=0}^{\infty} a^{n} e^{-(n+1)^{\lambda} s}\right]
$$

which leads to

$$
\begin{aligned}
G_{1}(\{x\} ; s)=\frac{1}{\left(1-x_{1}\right) \ldots\left(1-x_{k}\right)}\left\{1-\sum_{j=1}^{k} x_{j}[1\right. & \left.-\left(1-x_{j}\right) \sum_{n=0}^{\infty} x_{j}^{n} e^{-(n+1)^{\lambda} s}\right] \\
& \left.+\sum_{j>r} \sum_{j} x_{j} x_{r}\left[1-\left(1-x_{j} x_{r}\right)\right] \sum_{n=0}^{\infty}\left(x_{j} x_{r}\right)^{n} e^{-(n+1)^{\lambda} s}-\ldots\right\}
\end{aligned}
$$

which reduces to eq (9) when $\lambda=1$.

The method suggested in this note can be generalized to deal with any functional of the form $M_{j}\left(\varphi\left(n_{1}\right), \varphi\left(n_{2}\right), \ldots \varphi\left(n_{k}\right)\right)$ providing that $\varphi(n)$ is a monotone increasing function which tends to infinity with $n$. It can also be used to calculate Laplace transforms rather than generating functions.

(Paper 68B1-110) 